\title{
Investigation of IL-17A, IL-17F, IL-34 Levels of Patient with Multiple Sclerosis and Pseudotumor Cerebri
}

\author{
Hayriye Soytürk', [MD] \\ ORCID: 0000-0002-0000-3768
}

Şeyda Karabörk², [MD]

ORCID: 0000-0002-9026-4485

Şule Aydın Türkoğlu ${ }^{3}$, [MD]

ORCID: 0000-0001-8616-832X

'Bolu Abant Izzet Baysal University Faculty of Agriculture, Gölköy, Bolu, Turkey.

${ }^{2}$ Bolu Abant Izzet Baysal University Faculty of Medicine, Department of Medical Microbiology, Gölköy, Bolu, Turkey.

${ }^{3}$ Bolu Abant Izzet Baysal University Faculty of Medicine, Department of Neurology, Gölköy, Bolu, Turkey.

The manuscript has been presented in a congress as an oral presentation, at 54th National Neurology Congress, Between November 30 and December 6, 2018, Antalya, Turkey.

Corresponding Author: Hayriye Soytürk Bolu Abant Izzet Baysal University, Faculty of Agriculture Gölköy, Bolu, Turkey.

E-mail: hayriyeorallar@ibu.edu.tr

https://doi.org/10.32552/2021.ActaMedica.498

\section{reD ABSTRACT Ceer}

Objective: Multiple Sclerosis is an inflammatory demyelinating disease characterized by lymphocyte infiltration and demyelination of brain tissue and central nervous system.

Materials and Methods: This study aimed to evaluate the interleukin (IL-17A, IL-17F, and IL-34 cytokine) levels in the cerebrospinal fluid of Relapsing-Remitting Multiple Sclerosis $(n=23)$, radiologically isolated syndrome $(n=5)$ and pseudotumor cerebri $(n=15)$ cases. In this study, lumbar puncture cerebrospinal fluid obtained from the patients who were diagnosed with Multiple Sclerosis aged between 21-55. The PTC group included patients with pseudotumor cerebri aged 28-60 years. The levels of IL-17A, IL-17F, IL-34 cytokines were determined by ELISA kit.

Results: In this study, Among the studied cytokines in the cerebrospinal fluid samples of the patients, median (min-max) values of IL-17A for the Demyelinated group and pseudotumor cerebri group were 50 (7-257) $\mathrm{pg} / \mathrm{ml}$ and 2 (1-6) $\mathrm{pg} / \mathrm{ml}$ respectively, a statistically significant difference $(p<0.01)$ has been observed in between the two groups. Median (minmax) values of IL-17F for the Demyelinated group and pseudotumor cerebri group were $32(6-891) \mathrm{pg} / \mathrm{ml}$ and $2(1-3) \mathrm{pg} / \mathrm{ml}$ respectively, a statistically significant difference $(p<0.01)$ has been observed between the two groups. Median (min-max) values of IL-34 for Demyelinated group and pseudotumor cerebri group were 16 (4-197) pg/ml and 2 $(1-11) \mathrm{pg} / \mathrm{ml}$ respectively, a statistically significant difference $(\mathrm{p}<0.01)$ has been observed in between the two groups (Lower limit for the cytokine values have been determined as IL-17A: 3,93 pg/ml, IL-17F: 2,23 pg/ml, IL-34: 3,12 pg/ml). IL-34, was found to be high in Multiple sclerosis patients. This is important for the cerebral endothelial reaction in Multiple sclerosis.

Conclusion: The high levels of IL-34 in cerebrospinal fluid samples suggest that it may be a new treatment strategy and an adjunct cytokine in the diagnosis of neuroinflammatory and neurodegenerative diseases such as multiple sclerosis and demyelinating disease. More extensive studies are needed to determine whether IL-34 can be a marker in the return of the disease from radiologically isolated syndrome to clinical MS.

Keywords: Multiple sclerosis, pseudotumor cerebri, radiological isolated syndrome, cytokine, Interleukin-17a, Interleukin-17f, Interleukin-34.

Received: 24 August 2020, Accepted: 28 March 2021,

Published online: 20 June 2021 


\section{INTRODUCTION}

Multiple Sclerosis (MS) is an autoimmune and disease of the central nervous system (CNS) and may reveal itself with chronicle, inflammatory, episodic neurological deficits, as well as with a neuroanatomic localization [1,2]. Relapsingremitting multiple sclerosis (RRMS) is known to be the most common form. Oftentimes, neurological deficits and damages occur that get worse in time and result in the development of secondary progressive multiple sclerosis (SPMS) [3].

In recent years, MS has been defined as a radiologically isolated syndrome (RIS) in patients without a typical MS clinic, with normal examination findings, and with MS-like demyelinating lesions on cranial MRI imaging for any reason, when other causes are excluded [4]. While evaluating the diagnostic criteria for MS, clinical and magnetic resonance (MR) findings play an important role in differentiating the MS cases from all other diseases. Apart from these, studies on cerebrospinal fluid (CSF) are also in progress [5]. CSF, evaluation of oligoclonal bands (OCBs) pointing to immunoglobulin $\mathrm{G}(\mathrm{lgG})$ index sensitivity of $95 \%$ and specificity above $80 \%$ are among the preferred methods [6].

Cytokines and chemokines in the CSF are the potential indicators of MS activity. During the active and/or flare-up periods of MS, an increase in the proinflammatory cytokines and chemokines is observed while the antiinflammatory cytokine and chemokines decrease $[7,8]$. In MS, local inflammation, demyelination, and neurodegeneration are triggered by effector $T$ cells that move from the periphery to the CNS. Although there are much data on T helper (Th) cells known as helper $T$ cells when developing new strategies for treatment, the role of the pro-inflammatory Th cells subtypes involved in the pathogenesis of MS has not been fully understood $[9,10,11]$. Both Th1 and Th17 cells are known to be encephalitogenic but these two cells follow different paths to enter into the central nervous system (CNS). In a study in which experimental encephalomyelitis was formed, it has been observed that Th1 cells moved towards the spinal cord whereas Th17 cells penetrated the brain [12]. It is also known that secretion of cytokines (such as IL-17A, IL-17F, IL-21, and IL-22.) from Th1 (IL-2, IL-12, TNF-alfa, and IFN-gamma) and Th17 cells increase relatively more in MS patients.
Interleukin-34 (IL-34), which is known to have communication with Th17 cells, has lately been among the cytokines that have been emphasized. IL34 is a cytokine that was expressed by macrophages, endothelial cells, fibroblasts, hepatocytes, and even neurons, and that contributes to the secretion of IL-17 via stimulating Th17 cells $[13,14]$. Once the literature is reviewed, although the study examining the relationship between cytokines and MS is limited, serum cytokine levels were examined instead of CSF samples. However, the relationship between IL-34 and neurodegenerative diseases has been remarkable in recent years [15].

Pseudotumor cerebri (PTC), is a disease with unclear pathophysiology and is characterized by inflammatory changes in the neurodegenerative disruptions on the absorption of CSF [16]. Having been clinically characterized by headache, papilledema, vision loss, and pulsatile tinnitus, no revealing cause has been identified due to radiological monitoring and CSF examinations [17]. Several studies have been performed on cytokines, as a candidate biomarker, to leverage them during the progressing, healing, and even rapid diagnosis phases of neurodegenerative diseases like MS and PTC. However, there are very few cytokine studies where both MS and PTC are focused together, and especially the CSF is examined.

In this study, we are targeting to research three different cytokines that are interrelated and found in the CSF samples of MS and PTC cases. For this purpose, through the evaluation of IL-17A, IL-17F, and IL-34 cytokine levels in CSF of the MS and PTC cases under the medical observation of our clinic, tracking of IL-34 in CSF is intended. Especially in

neuroinflammatory and neurodegenerative diseases like MS, so that new strategy could be developed for diagnosis or treatment which can be used by the clinicians in the future.

\section{MATERIALS and METHODS}

For this research, authorization with the approval code of 2018/203 has been granted by the Local Ethics Committee. All procedures performed in studies involving human participants were under the ethical standards of the institutional and 
national research committee and with the 1964 Helsinki declaration and its later amendments or comparable ethical standards. This article does not contain any studies with animals performed by any of the authors.

A total of 43 cases have been included in this study. Two main groups were constituted, demyelinated and PTC. The demyelinating group was selected from the cases who admitted to the clinic, for CSF collection through a lumbar puncture (LP) for diagnostic purposes, whose CSF samples were stored at $-80^{\circ} \mathrm{C}$. Since none of the cases was diagnosed at the time of LP procedure, the RRMS or RIS diagnosis was made depending on whether there was an episode in the follow-up of these cases that were not under any MS treatment at the time of CSF collection.

Cases that were admitted to the Neurology Department of Bolu Abant Izzet Baysal University, Educational Research Hospital between February 2015 and February 2018 were used in this study where we had the RRMS group with ages varying between 21 and 55, who were diagnosed $(n=23)$ with RRMS based on McDonald criteria. The RRMS group consisted of patients who applied to the clinic at the first MS attack and had CSF taken by performing LP for diagnostic purposes. For this reason, CSF was taken during the attack in the RRMS group.The RIS group with ages varying between 20 and 65 who showed lesions similar to MS during cranial imaging but could not be diagnosed $(n=5)$ with MS based on McDonald criteria,

Patients who had MS-like lesions in cranial imaging taken for any reason, but did not have any episodes were defined as RIS and a PTC-diagnosed $(n=15)$ control group with ages varying between 18 and 58 . There are no known attacks in the RIS group. Via LP, CSF samples have been obtained from the approved individuals in the control and experimental groups and these samples have been stored at $-80^{\circ} \mathrm{C}$ until assayed. All the cases (persons) were informed about the LP procedure and their approvals were collected before performing LP on them. Before the LP procedure, we made sure none of the cases had an active systemic and CNS infection. Other factors (such as Cerebral Venous Thrombosis and Behcet's disease) that could increase the CSF pressure on PTC cases were eliminated. Age, sex, duration of the disease, clinical status, and standard biochemical blood, and CSF analyzes were also evaluated according to medical records.

\section{Cytokine Measurements}

Serum cytokine levels (IL-17A, IL-17F, and IL-34) were measured with an ELISA (Elabscience, USA, CloudClone Corp., USA) according to the manufacturer's instructions. Results were expressed in picograms per milliliter. The specificity and the sensitivity for the cytokines were defined according to the manufacturer's instructions (specificity: except in IL-17A cytokine measurements in which crossreactivity with human IL-17F was negligible, noncross-reactivity was observed). Statistical analysis of the data was performed in SPSS 17.0 Statistics software package. As a result, since the data came out to be the normal distribution, the effects of the conditions were tested using the KruskalWallis, which is a non-parametric test. Conditional differences were analyzed using the MannWhitney-U test $p<0.05$ was considered statistically significant.

\section{RESULTS}

The group, who was diagnosed with RRMS in the new-onset and subsequent follow-up without any treatment, the RIS group, which was compatible with MS as an MR lesion and did not describe an attack and had no clinic, and the PTC group were The age averages of the RRMS, RIS and PTC, 34 $( \pm 10), 42( \pm 17)$, and $41( \pm 12)$ respectively. There was a statistically significant difference between all groups, $(p=0.01)$. Once a subgroup analysis was performed using the Bonferroni method, a statistically significant difference $(p=0.01)$ has been revealed especially between the RRMS group and PTC group.

While there are 13 female and 15 male cases in the RRMS and RIS (demyelinated) groups, this ratio is 10 to 5 respectively in the PTC group, and there was no statistically significant difference $(p=0.2)$ from a gender perspective between the two groups. Demographical data is shown in Table 1. As a result of the CSF analysis, no cell was found in the CSF of any of the cases. There was no statistically significant difference between the biochemical values of the CSF of two groups in Table 2 .

Of the 23 patients followed-up with RRMS diagnosis, 15 patients was Type 2 positive, 5 patients had Type 1 negative, 2 patients had Type 4 positive, 1 patient had Type 3 positive OCBs result, and the $\lg \mathrm{G}$ index 
Table 1. Demographical data and clinical characteristics/features of the patients.

\begin{tabular}{|l|c|c|c|c|}
\hline \multirow{2}{*}{$\mathrm{n}=43$} & \multicolumn{2}{|c|}{ Demyelinating Disease } & \multirow{2}{*}{$\operatorname{PTC}(\mathrm{n}=15)$} & $\mathrm{p}$ \\
\cline { 2 - 5 } & $\mathrm{MS}(\mathrm{n}=23)$ & $\mathrm{RIS}(\mathrm{n}=5)$ & $41( \pm 12)$ & $0.1^{\circ}$ \\
\hline Age mean $( \pm \mathrm{St})$ & $34( \pm 10)$ & $42( \pm 17)$ & $10 / 5$ & $0.2^{*}$ \\
\hline
\end{tabular}

MS: Multipl Skleroz, RIS: Radiological Isolated Syndrome, PTS: Psödotümör Serebri

${ }^{*}$ Chi-Square ${ }^{\circ}$ Oneway-Anova (Bonferroni)

Table 2. Comparison of the biochemical values of CSF in between the groups.

\begin{tabular}{|l|c|c|c|c|c|c|c|c|}
\hline & CSF Protein & Serum Protein & CSF Albumin & Serum Albumin & CSF Glucose & Serum Glucose & CSF LDH & CSF Klor \\
\hline Case & 30 & 7 & 15 & 4 & 71 & 104 & 16 & 125 \\
& $(15-226)$ & $(6-71)$ & $(1-30)$ & $(4-10)$ & $(50-126)$ & $(98-110)$ & $(10-41)$ & $(119-131)$ \\
PTC & 36 & 7 & 16 & 4 & 63 & 107 & 18 & 124 \\
& $(17-174)$ & $(7-8)$ & $(1-30)$ & $(4-5)$ & $(54-83)$ & $(104-112)$ & $(10-31)$ & $(120-130)$ \\
$\mathrm{p} \pm=$ & 0.7 & 0.9 & 0.5 & 0.1 & 0.3 & 0.07 & 0.5 & 1 \\
\hline
\end{tabular}

Case: Demyelinating Group, PTC: Pseudotumor Cerebri, CSF: cerebrospinal fluid \pm Mann-Whitney-U

was measured as $0.97( \pm 0.4)$ on the average. OCB and IgG index were not measured in 3 of 5 RIS cases. 2 of them were found to be OCBs Type I. The index was 0.70 in 1 case and 0.56 in the other. All three levels of cytokines; A statistically significant difference was found between IL-17A, IL-17F, IL-34 RRMS and RIS cases $(p=0.002, p=0.001, p=0.001$, respectively). Especially in the CSF analysis of RRMS cases who were taken during the attack period, it was observed that the levels of all 3 cytokines were significantly high (table 3 ). In comparison of CSF cytokine levels of demyelinating group and PTS cases in which RRMS and RIS cases were evaluated together; Among the studied cytokines in the CSF samples of the patients, median (minmax) values of IL-17A for the Demyelinated group and PTC group were 50 (7-257) $\mathrm{pg} / \mathrm{ml}$ and 2 (16) $\mathrm{pg} / \mathrm{ml}$ respectively, a statistically significant difference $(p<0.01)$ has been observed in between the two groups. Median (min-max) values of IL-17F for the Demyelinated group and PTC group were $32(6-891) \mathrm{pg} / \mathrm{ml}$ and $2(1-3) \mathrm{pg} / \mathrm{ml}$ respectively, a statistically significant difference $(p<0.01)$ was observed between the two groups. Median (minmax) values of IL-34 for the Demyelinated group and PTC group were 16 (4-197) pg/ml and 2 (111) $\mathrm{pg} / \mathrm{ml}$ respectively, a statistically significant difference $(p<0.01)$ was observed between the two groups (Lower limit for the cytokine values have been determined as IL-17A: 3,93 pg/ml, IL-17F: 2,23 $\mathrm{pg} / \mathrm{ml}$, IL-34: 3,12 pg/ml) in Table 3 .

Levels of IL-17A and IL-17F, which are secreted by Th17 cells, as well as the levels of IL-34, that stimulate the secretion of IL-17 by Th17 cells, were determined in the CSF samples of the PTC and the Demyelinated disease groups. Throughout the study, a statistically significant increase in the levels of IL-34 (secreted by Th17 (IL-17A, IL-17F) and also by a variety of cells such as hearth, brain, liver, kidney, spleen, and thymus gland cells) was observed in the MS group concerning the PTC group. The difference in cytokine levels between the Demyelinated group and the PTC group was shown in Table 3.

\section{DISCUSSION}

MS is an inflammatory-demyelinating disease characterized by lymphocyte infiltration and demyelination of brain tissue and central nervous system. At the diagnostic stage, it is of quite an importance to examine the patient's clinical findings together with MRI imaging and CSF analysis [2]. Numerous studies are focusing on biomarker definitions in CSF and serum samples, cytokine and chemokine levels related to inflammation, and biochemical and microbial factors and investigating the roles of these factors in MS $[8,18]$. Autoimmune inflammation usually occurs in the early stages of MS, followed by the neurodegenerative phase. The degradation of the myelin occurs as a result of these changes.

It is believed that myelin-specific $T$ lymphocytes may have a role in the pathogenesis of the disease. Th1 and Th17 lymphocytes have been shown to 
Table 3. Comparison of CSF cytokine levels in between the groups.

\begin{tabular}{|c|c|c|c|}
\hline \multirow[t]{2}{*}{ Group $n=43$} & \multicolumn{2}{|c|}{ Demyelinating Disease $n=28$} & \multirow{2}{*}{$\mathrm{PTC}^{*} \mathrm{n}=15$} \\
\hline & RRMS $^{*} n=23$ & RIS $^{*} n=5$ & \\
\hline \multirow{4}{*}{$\begin{array}{l}\text { IL-17A (pg/ml) } \\
\text { median (min-max) }\end{array}$} & $59(7-257)$ & $20(10-22)$ & \\
\hline & \multicolumn{2}{|c|}{$p=0.002$} & \\
\hline & \multicolumn{2}{|c|}{$50(7-257)$} & $2(1-6)$ \\
\hline & & $p<0.001^{\circ}$ & \\
\hline \multirow{5}{*}{$\begin{array}{l}\text { IL-17F (pg/ml) } \\
\text { median (min-max) }\end{array}$} & RRMS $^{*} \mathrm{n}=23$ & $\mathrm{RIS}^{*} \mathrm{n}=5$ & PTC $^{*} n=15$ \\
\hline & $36(15-891)$ & $8(6-15)$ & \\
\hline & \multicolumn{2}{|c|}{$p=0.001$} & \\
\hline & \multicolumn{2}{|c|}{$32(6-891)$} & $2(1-3)$ \\
\hline & & $p<0.001^{\circ}$ & \\
\hline \multirow{5}{*}{$\begin{array}{l}\text { IL-34 (pg/ml) } \\
\text { median (min-max) }\end{array}$} & RRMS $^{*} \mathrm{n}=23$ & RIS $^{*} n=5$ & PTC $^{*} n=15$ \\
\hline & $23(7-197)$ & $5(4-9)$ & \\
\hline & \multicolumn{2}{|c|}{$p=0.001$} & \\
\hline & \multicolumn{2}{|c|}{$16(4-197)$} & $2(1-11)$ \\
\hline & & $p<0.001^{\circ}$ & \\
\hline
\end{tabular}

*RRMS: Relapsing Remitting Multiple Sclerosis *RIS: Radiologically Isolated Syndrome *PTC: Pesudotumor cerebri ${ }^{\circ}$ Mann-Whitney U

play a role in the pathogenesis of MS. A significant increase in the number of Th17 cells was observed during the exacerbation of MS [19]. Many studies are showing the association of MS with IL-17A and IL-17F synthesized by Th17 cells [18,20,21].

IL-17 has been shown to disrupt the integrity of the blood-brain barrier in both primary microvascular endothelial cells [22] taken from the human brain and in mouse brain endothelial cell line [23]. IL17 has been shown to play a role in the formation of experimental autoimmune encephalomyelitis (EAE). The blood-brain barrier (BBB) is less impaired and disease scores were lower in mice lacking IL17A with IL-EAE [23]. Anti-IL17A treatment has been shown to reduce BBB degradation in elderly wild-type C57BL/6J mice [24].

In experimental studies, IL-17A blockade has been shown to weaken autoimmune encephalomyelitis (EAE). Also, it is elevated in CSF of RRMS patients and associated with CSF/serum albumin (Qalb), a measure of blood-brain barrier (BBB) dysfunction. In the cell culture studies, the combination of IL$17 \mathrm{~A}$ and IL- 6 has also been shown to reduce the expression of genes associated with tight junction (TJ) proteins in the blood-brain barrier cell line (hCMEC/D3) and impair monolayer integrity. In CSF of RRMS patients, unlike cell culture studies, no correlation was found between IL-6 and Qalb, while IL-17A increases. IL-17A rises in CSF of RRMS patients, but IL-17F is low in these cases. CSF IL-
17A levels are associated with blood-brain barrier damage in RRMS. A combination of IL-17A and IL- 6 impairs endothelium tight-link gene expression and monolayer integrity. These studies show that the targeting of IL-17A is of potential importance in maintaining blood-brain barrier integrity in RRMS [25].

Although it was shown that IL-17A was high and IL$17 F$ was low in RRMS patients, this study showed that both values were higher in RRMS and RIS patients than in PTC patients. Moreover, when we look at the CSF/Serum albumin ratio, there are no significant differences between the RRMS and PTS groups.

IL-34 plays a role in the development of the nervous system $[26,27]$. CSF-1 is structurally expressed in the ventricular and subventricular regions of the brain [28]. In mice with IL-34 deficiency, the decrease in microglial cells in the hippocampus and cortex proves the role of IL-34 in microglia development and homeostasis [29]. Interleukin (IL) -34 is a recently discovered cytokine, identified as a ligand of the first colony-stimulating factor (CSF) - 1 receptor (CSF-1R).IL-34 is involved in a wide variety of signal pathways and biological functions. In physiological conditions, IL-34 expression has a fundamental role in cellular differentiation, adhesion and migration, and proliferation. Studies are showing that IL-34 can play quite an important role in the development of microglia and Langerhans cells $[28,30]$. 
IL-34 is a cytokine produced by fibroblasts in the brain, T cells, neurons, and keratinocytes in the skin; it also binds the macrophage colony-stimulating factor 1 receptor (m-CSF-1). IL-34 is also known to bind colony-stimulating factor 1 (CSF-1). Studies are showing that $T$ reg cells, known as regulatory T cells, also produce IL-34 [15]. IL-34 has a proinflammatory effect in humans and can contribute to inflammation in various diseases. Moreover, IL-34 is stimulated by pathogen-associated molecular patterns (PAMP) and inflammatory cytokines [20]. Serum IL-34 levels have been reported in hepatitis $B$ and rheumatoid arthritis (RA) patients who develop liver fibrosis, patients with acute rejection after liver transplantation in patients, and in various diseases, including systemic lupus erythematosus (SLE), periodontal disease, and cancer. Although the potential role of IL-34 in these diseases is still unclear, it is known that IL-34 is an immunoregulatory cytokine due to its inflammatory and immunosuppressive properties [21].

IL-34 may be useful as a therapeutic target in the control of diseases. IL-34 can prevent various diseases such as neurological disorders, skin lesions, transplant rejection, and infections. IL-34 provides powerful neuroprotection through its action on microglia, neurons, and endothelial cells. It can cause pluripotent stem cells to differentiate into microglia, suggesting a potential role in neural regeneration [31].

IL-34 also regulates the microglial proliferation by inducing the expression of transforming growth factor-beta (TGF- $\beta$ ) in microglia; moreover, IL-17 concentration has been shown to increase as a result of excitation of and peripheral mononuclear cells in RA patients [21].

The role of IL-34 and the relationship of IL-17 in MS are summarized in Figure 1. Due to these correlations of IL-34 with Th17 cytokines, it was hypothesized that levels of IL-34 and IL-17 in MS patients may differ from PTC. We evaluated IL-17A, IL-17F, and IL-34 levels in CSF samples of MS patients with RIS symptoms according to McDonald's criteria and PTC patients as the control group.

In our study, a statistically significant difference was found in both IL-17A, IL-17F and IL-34 cytokine levels in CSF samples of MS patients compared to the PTC group $(p<0.001)$. In line with the literature, the results of this study suggest that IL-34 can be upregulated in the pathogenesis of RA, Hepatitis $B, S L E, M S$, and even other neurodegenerative diseases.

The most important limitation is that this study was carried out retrospectively on CSF samples stored at $-80^{\circ} \mathrm{C}$. Due to ethical issues related to

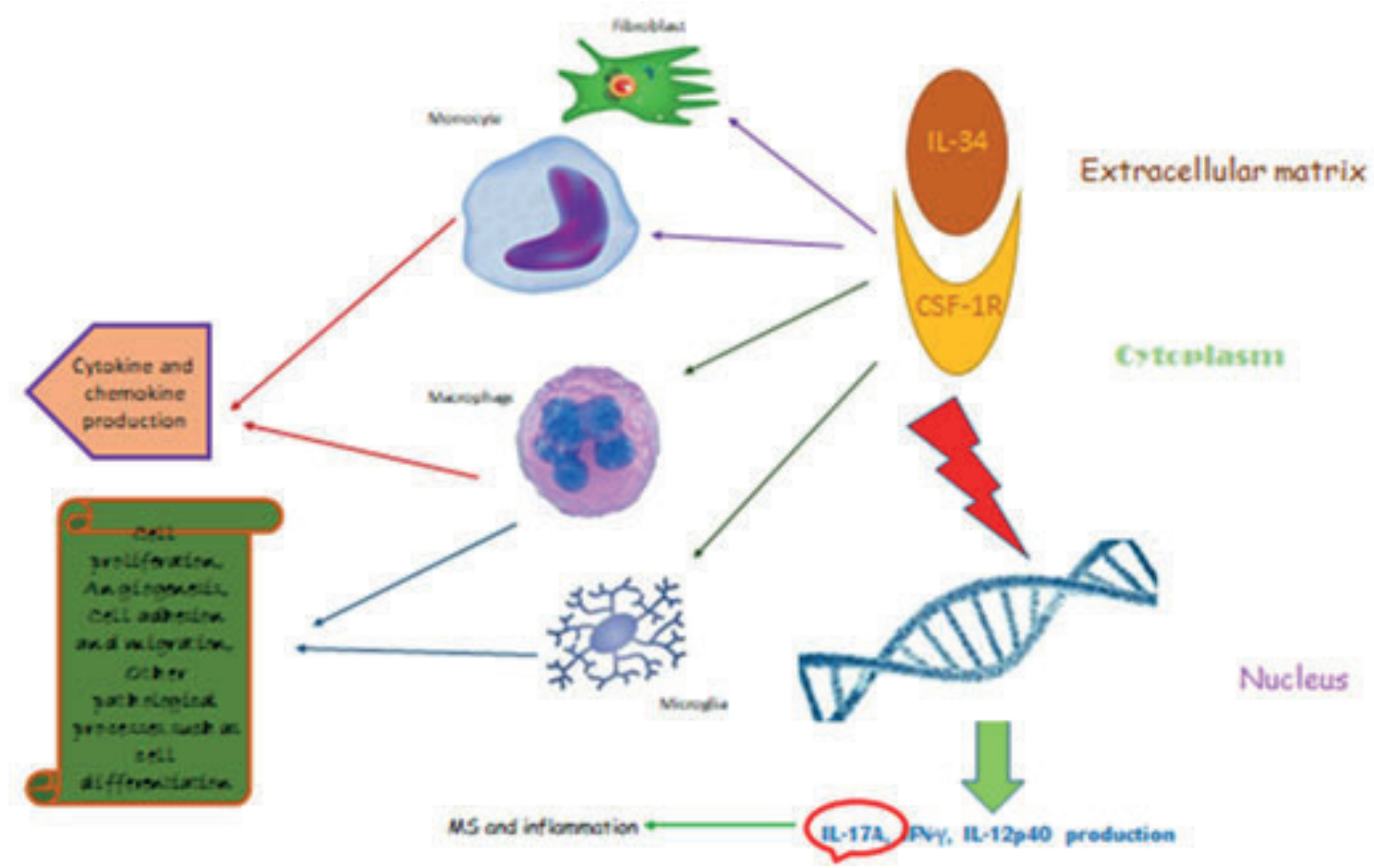

Figure 1. The role of IL-34 in multiple sclerosis and the relationship between IL-17 (Figure 1 is drawn by the author (Karabork S)). 
CSF sample collection from healthy individuals, the control group consisting of PTC patients, instead of normal healthy individuals, is another limitation.

In this study, IL-17, which is known to play a role especially in autoimmune diseases, showed an increase in RRMS patient group CSF samples compared to PTC group CSF samples. It is also noteworthy that IL-34, a new cytokine known to be expressed from neurons in the CNS, is high in RRMS patients. This novel function of IL-34 and its elevated levels in CSF samples suggest that it may be used as a novel treatment strategy and/ or an adjunct cytokine in neuroinflammatory and neurodegenerative diseases, such as Alzheimer's and MS, and may contribute to this aspect of literature.

It is also noteworthy to state that these interleukin levels are high in RIS, which fails to be diagnosed with MS according to Mc Donald's diagnostic criteria, but is characterized by demyelinating lesions. More comprehensive studies are needed to determine whether IL-34 is a marker in the disease's transition from RIS to clinical Ms.
As a result, this study will contribute to the early diagnosis and treatment of MS, a neurodegenerative disease that is difficult and not yet possible to diagnose early. IL-34 and IL-17A are promising both as a biomarker for the diagnosis of disease and a therapeutic agent that can be used in the treatment of various diseases in the clinic. More comprehensive, further research is needed to clarify this issue. Specifically, determining IL-34, IL-17A, and IL-17F CSF levels, then diagnosing MS, and identifying biochemical processes involving this cytokine may contribute to the development of precise treatment strategies for MS.

\section{ACKNOWLEDGMENT}

The authors would like to acknowledge the patients and their families.

\section{CONFLICT of INTEREST}

No potential conflict of interest was reported by the authors.
[1] Compston A, Coles A. Multiple sclerosis. Lancet (London, England). 2008; 372(9648): 1502-1517.

[2] Thompson AJ, Banwell BL, Barkhof F, et al. Diagnosis of multiple sclerosis: 2017 revisions of the McDonald criteria. Lancet Neurol. 2018; 17(2): 162-173.

[3] Bjartmar C, Trapp BD. Axonal and neuronal degeneration in multiple sclerosis: mechanisms and functional consequences. Curr Opin Neurol. 2001; 14(3): 271-278.

[4] Okuda DT, Mowry EM, Beheshtian A, et al. Incidental MRI anomalies suggestive of multiple sclerosis: the radiologically isolated syndrome. Neurology. 2009; 72(9): 800-805.

[5] Andersson M, Alvarez-Cermeño J, Bernardi G, et al. Cerebrospinal fluid in the diagnosis of multiple sclerosis: a consensus report. J Neurol Neurosurg Psychiatry.1994; 57(8): 897-902.

[6] Dendrou CA, Fugger L, Friese MA. Immunopathology of multiple sclerosis. Nat Rev Immunol. 2015; 15(9): 545-558.

[7] Dargahi N, Katsara M, Tselios T, et al. Multiple Sclerosis: Immunopathology and Treatment Update. Brain Sci. 2017; 7(7): 78.
[8] Van Oosten BW, Lai M, Hodgkinson S, et al. Treatment of multiple sclerosis with the monoclonal anti-CD4 antibody CM-T412: results of a randomized, double-blind, placebocontrolled, MR-monitored phase II trial. Neurology.1997; 49(2): 351-357.

[9] Mahad DH, Trapp BD, Lassmann H. Pathological mechanisms in progressive multiple sclerosis. Lancet Neurol. 2015; 14(2): 183-193.

[10] Van Langelaar J, Van der Vuurst de Vries RM, Janssen M, et al. T helper 17.1 cells associate with multiple sclerosis disease activity: perspectives for early intervention. Brain. 2018; 141(5): 1334-1349.

[11] Stromnes IM, Cerretti LM, Liggitt D, et al. Differential regulation of central nervous system autoimmunity by $\mathrm{T}(\mathrm{H}) 1$ and $\mathrm{T}(\mathrm{H}) 17$ cells. Nat Med. 2008; 14(3): 337-342.

[12] Baghdadi M, Umeyama Y, Hama N, et al. Interleukin-34, a comprehensive review. J Leukoc Biol. 2018; 104(5): 931-51.

[13] Sospedra, M, Martin, R. Immunology of Multiple Sclerosis. Semin Neurol. 2016; 36(2): 115-127.

[14] Truong AD, Hong Y, Lee J, et al. Interleukin-34 Regulates Th1 and Th17 Cytokine Production by Activating Multiple Signaling Pathways through CSF-1R in Chicken Cell Lines. Int J Mol Sci. 2018; 19(6): 1665. 
[15] Portelli M, Papageorgiou PN. An update on idiopathic intracranial hypertension. Acta Neurochir (Wien). 2017; 159(3): 491-499.

[16] Friedman DI. The pseudotumor cerebri syndrome. Neurol Clin. 2014; 32(2): 363-396.

[17] Matejčíková Z, Mareš J, Sládková V, et al. Cerebrospinal fluid and serum levels of interleukin-8 in patients with multiple sclerosis and its correlation with Q-albumin. Mult Scler Relat Disord. 2017; 4: 12-15.

[18] Brucklacher-Waldert V, Stuerner K, Kolster M. Phenotypical and functional characterization of $\mathrm{T}$ helper 17 cells in multiple sclerosis. Brain. 2009; 132(Pt 12): 3329-3341.

[19] Kebir H, Ifergan I, Alvarez Jl. Preferential recruitment of interferon-gamma-expressing $\mathrm{TH} 17$ cells in multiple sclerosis. Ann Neurol. 2009; 66(3): 390-402.

[20] Xie HH, Shen $H$, Zhang $L$, et al. Elevated Serum Interleukin-34 Level in Patients with Systemic Lupus Erythematosus Is Associated with Disease Activity. Sci Rep. 2018; 8(1): 3462.

[21] Tian Y, Shen H, Xia L, et al. Elevated serum and synovial fluid levels of interleukin-34 in rheumatoid arthritis: possible association with disease progression via interleukin-17 production. J Interferon Cytokine Res. 2013; 33(7): 398-01.

[22] Rahman MT, Ghosh C, Hossain M, et al. IFN-gamma, IL-17A, or zonulin rapidly increase the permeability of the bloodbrain and small intestinal epithelial barriers: relevance for neuro-inflammatory diseases. Biochem Biophys Res Commun. 2015; 129: 625-637.

[23] Huppert J, D. Closhen, A. Croxford, et al. Cellular mechanisms of IL-17-induced blood-brain barrier disruption. FASEB J. 2010; 24(4): 1023-34.
[24] Ni P, Dong H, Wang $Y$, et al. IL-17A contributes to perioperative neurocognitive disorders through blood-brain barrier disruption in aged mice $\mathrm{J}$. Neuroinflammation.2018;15: 332

[25] Setiadi FA, Abbas AR, Jeet WK, et al. IL-17A is associated with the breakdown of the blood-brain barrier in relapsingremitting multiple sclerosis. J Neuroimmunol.2019; 332: 147-154.

[26] Felix J, Elegheert J, Gutsche I, et al. Human IL-34 and CSF-1 establish structurally similar extracellular assemblies with their common hematopoietic receptor. Structure. 2013; 21: 528-539.

[27] Nakamichi Y, Udagawa N, Takahashi N. IL-34 and CSF-1: similarities and differences. J Bone Miner Metab. 2013; 31: 486-495.

[28] Wang Y, Szretter KJ, Vermi W, et al. IL-34 is a tissuerestricted ligand of CSF1R required for the development of Langerhans cells and microglia. Nat Immunol. 2012; 13: 753-760.

[29] Wang Y, Colonna M. Interkeukin-34, a cytokine crucial for the differentiation and maintenance of tissue resident macrophages and Langerhans cells. Eur J Immunol.2014; 44:1575-1581

[30] Greter M, Lelios I, Pelczar P, et al. Stroma-derived interleukin-34 controls the development and maintenance of Langerhans cells and the maintenance of microglia. Immunity. 2012; 37:1050-1060

[31] Ge Y, Huang M, Yao Ym. Immunomodulation of Interleukin-34 and its Potential Significance as a Disease Biomarker and Therapeutic Target. Int J Biol Sci 2019; 15(9):1835-1845. 